\title{
DE NUEVO SOBRE EL DESISTIMIENTO EN DERECHO PENAL. A PROPÓSITO DE LAS CUATRO REGLAS SOBRE LA VOLUNTARIEDAD DE RESTREPO RAMOS*
}

Diego Araque Moreno**

\begin{abstract}
Resumen: El presente artículo trata sobre el requisito de la voluntariedad del desistimiento de consumar la conducta punible, examinando a tales efectos las distintas propuestas que se suscitan entre las teorías psicológicas y normativas, optando el autor por una posición mixta que se desarrolla a partir de los criterios de libertad de voluntad y ausencia de coacción como causa de inculpabilidad penal.
\end{abstract}

\footnotetext{
El presente artículo se desarrolló en el marco del proyecto de investigación "Principios y garantías penales: sectores de riesgo", cuyo investigador principal es el Prof. Dr. Miguel Díaz y García Conlledo de la Universidad de León, España, y de cuyo equipo de trabajo hago parte. El proyecto es financiado por el Ministerio de Economía y competitividad de España (Mineco, DER2016-76715-R).

** Abogado y especialista en derecho penal de la Universidad de Antioquia. Profesor de Derecho Penal, Universidad de Medellín, Colombia. Correo-e: diegoaraque1973@yahoo.com. Fecha de recepción: 24 de octubre de 2016. Fecha de modificación: 15 de noviembre de 2016. Fecha de aceptación: 24 de febrero de 2017. Para citar el artículo: Araque Moreno, Diego. "De nuevo sobre el desistimiento en derecho penal. A propósito de las cuatro reglas sobre la voluntariedad de Restrepo Ramos", en Revista Derecho Penal y Criminología, Vol. 37, n. ${ }^{\circ}$ 103, julio-diciembre de 2016, Bogotá, Universidad Externado de Colombia, pp. 13-34, Dor: https://doi.org/10.18601/01210483.v37n103.02
} 
Palabras clave: tentativa, consumación, desistimiento, voluntariedad, libertad de voluntad, coacción.

\title{
BACK ON WITHDRAWAL IN CRIMINAL LAW. A PURPOSE OF THE FOUR RULES ON THE ACCEPTANCE OF RESTREPO RAMOS
}

\begin{abstract}
This article deals with the requirement of voluntariness of withdrawal of consummating the criminal offense, examining such effects the various proposals that arise between psychological theories and regulations, choosing the author by a joint position that develops from the criteria of freedom of will and absence of coercion as a cause of criminal culpability.

Keywords: attempted; consummation; withdrawal; willfulness; freedom of will; coercion.
\end{abstract}

\section{INTRODUCCIÓN}

En el año 2008 publiqué una pequeña obra sobre la figura jurídica del desistimiento en Derecho penal, entendido este como la eficaz evitación del resultado típico llevada a feliz término por la propia voluntad del agente y que por ello mismo genera unas consecuencias jurídicas de tipo sustancial, la principal de ellas, la impunidad en relación con la conducta punible con respecto a la cual efectivamente se desiste. En dicha ocasión me ocupé de varios temas relacionados con el problema en cuestión, a saber, el fundamento del desistimiento, su ubicación sistemática dentro de la teoría del delito, sus requisitos, consecuencias jurídicas y su regulación en el Código Penal colombiano ${ }^{1}$. Uno de los problemas allí tratados estaba relacionado con el requisito de la voluntariedad. Es necesario que sea voluntario el desistimiento para que exonere de responsabilidad penal.

A estos efectos, piénsese en el siguiente caso sometido a consideración de la Magistratura colombiana y resuelto en casación de 17 de marzo de 2004 por la Corte Suprema, Sala Penal, mediante radicado 13.171: un sujeto, después de una discusión con su prometida, producto de la ingesta de licor y de una situación de celos, en el apartamento de esta y en horas de la madrugada, le propinó un disparo en la cabeza, y se quedó dormido allí mismo instantes posteriores a la realización del hecho. En horas de la mañana, luego de despertar y advertir que la joven todavía estaba con vida, salió de la casa de esta y, prevalido de un vehículo de servicio público, la trasladó a un centro asistencial donde le fue salvada la vida. La pregunta a resolver entonces viene dada por si en estos hechos puede considerarse como voluntario el acto de desistimiento.

1 Araque Moreno, Diego (2008). 
De un caso próximo se ocuparon los tribunales alemanes, del cual da cuenta Roxin en los siguientes términos:

A, con intención de matarla, lesionó a la víctima poniendo en peligro su vida, pero llamó a continuación a un médico, que salvó a la víctima. Según la conclusión del juez del caso, la contemplación de la mujer lesionada fue “demasiado para él (...), simplemente, él ya no pudo más', 'no quería ya que la mujer se desangrara en el sótano" 2 .

De nuevo: ¿puede considerarse que en este otro evento el autor desistió de forma voluntaria?

En esta nueva oportunidad me ocuparé una vez más de este aspecto en particular, esto es la voluntariedad en el desistimiento de consumar la conducta punible. En esta ocasión lo haré como consecuencia de un trabajo recientemente publicado en esta misma Revista por mi colega Jorge C. Restrepo Ramos, en el cual se abordó esta misma problemática ${ }^{3}$. Procuraré por consiguiente profundizar sobre el requisito de la voluntariedad del desistimiento en materia penal a efectos de poner a prueba algunas de las hipótesis del trabajo últimamente referido y desde luego desarrollar mi propia postura sobre el particular.

En consecuencia, dejaré de lado los otros problemas que se generan en relación con la figura jurídica del desistimiento, en relación con los cuales remito a mi trabajo del año 2008.

Con este panorama, me ocuparé en principio de ubicar la discusión dentro de la sistemática de la teoría del delito. A continuación procederé a concretar el problema de la voluntariedad en materia de desistimiento penal. Seguidamente, desarrollaré mi posición en el sentido de que la voluntariedad debe ser valorada como expresión de ausencia de cualquier evento de coacción. En cuarto lugar llevaré a cabo algunas consideraciones en relación con la contribución de Restrepo Ramos, con quien coincido en los resultados como se tendrá oportunidad de ver en su momento, aunque por razones metodológicas distintas. Por último, expondré mis propias conclusiones.

\section{UBICACIÓN SISTEMÁTICA DEL PROBLEMA}

Con independencia de la fundamentación sistemática del delito que se asuma, esto es, una posición causal, finalista o funcionalista, es claro que la realización de una conducta punible puede quedar en fase de tentativa. Esto es algo que reconocen por

2 Roxin, Claus (2014, p. 720).

3 Restrepo Ramos, Jorge C. (2015, pp. 153 y ss.). 
igual las distintas posturas metodológicas de construcción racional del delito. Ello sucederá en términos generales cuando el resultado típico no se produzca por razones ajenas a la voluntad del autor. En estos casos se habla entonces de la existencia de un delito tentado o imperfecto, de un conato delictivo, por oposición a la consumación o perfección plena de la realización típica, esto es, cuando el resultado típico en cambio sí se produce o se alcanza en el caso particular. No corresponde al presente trabajo profundizar sobre el tema y los no poco problemas que se generan a propósito del dispositivo amplificador del tipo penal de la tentativa. Para ello remito, asimismo, a otro de mis trabajos en el cual he desarrollado algunos de estos aspectos ${ }^{4}$.

Además de los dos eventos anteriormente señalados, valga recordar, tentativa y consumación, es posible que el resultado típico no se produzca por circunstancias propias a la voluntad del mismo autor que con su conducta previa creó un riesgo jurídicamente desaprobado para el respectivo bien jurídico. En este otro supuesto se reconoce por parte de la doctrina y la jurisprudencia la configuración del fenómeno jurídico del desistimiento de consumar la conducta punible, cuyo principal efecto -como ya se manifestó- será la impunidad con respecto al delito por el cual se desiste. "La institución o la figura jurídica del desistimiento o del retroceso en la consumación de la conducta punible, se presenta cuando el agente, a pesar de haber dado comienzo a los actos de ejecución -esto es, aquellos que demarcan o delimitan el campo entre lo que está permitido y lo que está prohibido en materia penal, y con los cuales el delito está ya tentado-, voluntariamente, por sí mismo, impide que se produzca el resultado en un comienzo querido, para lo cual basta con la simple o mera interrupción de su propósito criminal en el caso de la tentativa simple o inacabada, ora mediante un hacer que contrarreste, anule o enerve lo realizado en tratándose de la denominada tentativa concluida o acabada" ${ }^{\text {. En los }}$ dos ejemplos de que se dio cuenta en la introducción del presente trabajo, deberá establecerse en primer lugar si la inconsumación -la no producción del resultado típico de la muerte- se explica por razones ajenas o propias a la voluntad del victimario. Si lo primero, habrá sanción penal a título de tentativa de homicidio. Si lo segundo, dándose los requisitos de la voluntariedad y la eficacia, no será posible la imposición de sanción alguna por el punible de tentativa, pues en este caso se configura entonces la institución jurídica del desistimiento (quedará intacta desde luego la sanción por el llamado delito remanente, esto es, para los casos propuestos, las lesiones finalmente ocasionadas).

Dos son por lo tanto los requisitos que exigen la doctrina y jurisprudencia dominantes de cara a reconocer los efectos de la impunidad penal en materia de desistimiento. Por

4 Araque Moreno, Diego (2011). En el mismo sentido puede verse el aporte de Córdoba Angulo, Miguel (2002, pp. 289-300). Más recientemente, quien quiera profundizar sobre los distintos temas de la tentativa podrá acudir al completísimo estudio de REYES Alvarado, Yesid (2016).

5 Araque Moreno, Diego (2008). 
un lado, que es justo el tema del presente trabajo, es necesario que el desistimiento sea voluntario. Por otro, se requiere que sea asimismo eficaz. Ambos requisitos obviamente deben concurrir para alcanzar los efectos de liberación de pena.

Ahora bien, en algunos casos el propio legislador se ha encargado de regular de lege lata la figura jurídica en cuestión y desde luego sus exigencias o requisitos correspondientes. Así por ejemplo, en el StGB (Código Penal alemán) el desistimiento se halla expresamente regulado en el parágrafo 24 , párrafo 1 , inciso 1 . Allí se dice que "no se castigará por tentativa a quien voluntariamente cesare en la ulterior ejecución del hecho o impidiere voluntariamente su consumación". En un camino próximo se encuentra el Código Penal español, en el cual se reglamentó el desistimiento, en el artículo 16.2, en los siguientes términos: "Quedará exento de responsabilidad penal por el delito intentado quien evite voluntariamente la consumación del delito, bien desistiendo de la ejecución ya iniciada, bien impidiendo la producción del resultado, sin perjuicio de la responsabilidad en que pudiera haber incurrido por los actos ejecutados, si estos fueren ya constitutivos de otro delito o falta".

Si bien el Código Penal colombiano no contempla alguna disposición que de forma expresa se ocupe del desistimiento de la consumación, de la redacción del inciso primero del artículo 27 que desarrolla la tentativa se puede colegir, mediante interpretación sensu contrario, las mismas exigencias de ley que se consagran en cambio en los códigos penales de Alemania y España, esto es, la voluntariedad y la eficacia. Lo anterior, dicho en otros términos: en el caso colombiano solo se puede llegar al desistimiento en materia penal y a sus requisitos en clave meramente interpretativa, deduciéndolos de la regulación del dispositivo amplificador que de la tentativa se realiza en el artículo 27 del Código Penal. Según este, habrá tentativa en aquellos casos en los cuales el autor inicia "la ejecución de la conducta punible mediante actos idóneos e inequívocamente dirigidos a su consumación, y esta no se produjere por circunstancias ajenas a su voluntad". De allí que no es posible hablar de tentativa en aquellos casos en los cuales el resultado típico no se produce pero por circunstancias propias, pues si ello sucede así simplemente la conducta o el comportamiento no se acoplará (atipicidad penal) a lo normado en el artículo 27 en cuestión. En estos eventos se configura entonces el desistimiento, en los cuales es el mismo autor el que ha neutralizado el riesgo para el respectivo bien jurídico, evitando de manera voluntaria la producción del resultado ${ }^{6}$.

6 Véase, sobre el desistimiento como causa de atipicidad penal objetiva en el Código Penal colombiano, Fernández Carrasquilla, Juan (2012,p. 775) y Córdoba Angulo, Miguel (2001, p. 118). Por mi parte, he dicho que el desistimiento en la legislación penal colombiana también puede ser interpretado como un problema de merecimiento de pena. Cfr. Araque Moreno, Diego (2009, pp. 93-109). 


\section{EL PROBLEMA: ¿CÓMO SE DETERMINA LA VOLUNTARIEDAD EN EL DESISTIMIENTO?}

El desistimiento de consumar la conducta punible, como se acabó de señalar, es considerado por doctrina y jurisprudencia como una causa de exoneración de responsabilidad penal. Ello, más allá de la discusión que existe en el sentido de si la persona que de forma voluntaria y eficaz desiste de la consumación deja sin efecto cualquiera de los presupuestos de imputación de responsabilidad penal (acción, tipicidad, antijuridicidad o culpabilidad) o si en estos casos se trata más bien de una especie de excusa legal absolutoria, personal de supresión o de necesidad de la pena. En el primer supuesto son razones de orden sistemático, de rigor dogmático o de merecimiento de pena las que impiden la configuración del delito. En el segundo evento, son motivos que en nada afectan la estructuración del injusto culpable y que en cambio están relacionados con el tema de la necesidad de pena ${ }^{7}$.

Independientemente de la ubicación del desistimiento dentro de la sistemática de la teoría del delito ${ }^{8}$, lo cierto es que este produce unos efectos jurídicos en materia penal. Como ya se ha dicho, la principal consecuencia de tipo jurídico será la impunidad del delito por el cual la persona desiste de su consumación o retrocede en su inicial propósito criminal. Para estos efectos se requiere sin embargo que el desistimiento cumpla con dos exigencias: voluntariedad y eficacia. "La impunidad del desistimiento (...) supone la anulación del fundamento de extensión de la pena, debiendo anular tanto el fundamento subjetivo como el objetivo. La anulación del primer fundamento se da con la voluntariedad del desistimiento, la del segundo con la evitación de la consumación del delito", señala Muñoz Conde desde una perspectiva que entiende el desistimiento como un problema de necesidad de pena ${ }^{9}$. Esto es así, se insiste, tanto si se considera que el desistimiento es un evento que excluye cualquiera de los presupuestos de imputación de responsabilidad penal, o bien una excusa legal absolutoria o, como Muñoz Conde y otros consideran, un supuesto de necesidad de pena.

El primer requisito del desistimiento en este orden de ideas consiste en que la persona que ha dado inicio a la ejecución de la conducta punible abandone o decline en su propósito criminal de manera completamente voluntaria. Es decir, el mismo sujeto que ha creado el riesgo jurídicamente desaprobado para el bien jurídico debe

7 Sobre el particular, cfr., Araque Moreno, Diego (2008, pp. 73-95).

8 En el caso colombiano, como ya lo dije, la posición dominante es aquella que considera que el desistimiento se erige en una causa de atipicidad objetiva. Esto se sostiene a partir de la interpretación que en sentido contrario se realiza del artículo 27 del Código Penal colombiano que consagra la figura de la tentativa, afirmándose que en los casos de desistimiento, en los cuales el resultado no se produce por circunstancias propias a la voluntad del autor, la conducta no se acopla al artículo en cuestión. Véase nota al pie 6.

9 Muñoz Conde, Francisco (1972, pp. 136 y 137). 
ser quien de forma voluntaria desactive el riesgo en cuestión y evite por lo tanto su realización en el resultado.

El problema radica entonces en establecer en qué casos el desistimiento es voluntario o no. Otro ejemplo, tomado de la literatura alemana: el sujeto comienza el ataque a la víctima, a la cual pretende acceder carnalmente contra su voluntad. Luego de que ha dado inicio a los actos de violencia, la mujer, sagazmente, con la esperanza de hallar la ayuda de un tercero, le dice al autor que no es necesario el uso de aquella, que ella puede acceder a sus pretensiones sexuales sin que sea necesario utilizar violencia alguna. El sujeto cesa en su propósito criminal y, con posterioridad, dos personas aparecen en el lugar de los hechos, lo cual es aprovechado por la mujer para solicitar su socorro provocando así la huida del lugar por parte del atacante. ¿Hay voluntariedad en este otro caso?

De la respuesta que se suministre a la anterior pregunta depende que se satisfaga este primer requisito de la voluntariedad del desistimiento de la consumación o del perfeccionamiento de la figura delictiva que en principio quería realizar el autor del hecho. Al respecto, "sigue existiendo, hoy como ayer, una controversia no resuelta sobre la determinación de la voluntariedad”, según señala Roxin sobre este punto en particular ${ }^{10}$.

Para la solución de este tipo de problemas se han elaborado distintas teorías. La discusión se suscita entre las teorías psicológicas y las normativas. Para las primeras la voluntariedad del desistimiento está condicionada a la autonomía o decisión propia del autor ${ }^{11}$. Por ello existirá voluntariedad en los dos primeros casos de que se ha dado cuenta en el presente trabajo (el autor motu proprio acude o solicita la intervención médica mediante la cual se termina salvando a la víctima), no así en el tercero (las personas que concurren al lugar del ataque sexual son las que obligan al victimario al retiro del lugar). Para las segundas, valga señalar las teorías normativas, el asunto depende de criterios de valoración o interpretación del suceso en sí mismo con miras a determinar el requisito de la voluntariedad examinado desde el criterio de retorno a la legalidad o al camino del Derecho y, por consiguiente, el respectivo examen del caso concreto a partir del tema de la necesidad de pena (misma solución a la propuesta en los tres casos por la postura psicológica pero por razones diferentes:

10 Roxin, Claus (2014, p. 714).

11 Adhieren a una teoría psicológica, entre otros, JESCHECK, HANS-HEINRICH Y WEIGEND, THOMAS (2002, pp. 584-585); y Maurach, Gössel y Zipf (1995, p. 106), en Alemania. En Colombia parten de esta posición Velásquez V., Fernando (2009, p. 971); Gómez LóPez, Jesús Orlando (2003, pp. 12061207); Fernández Carrasquilla, Juan (2012, pp. 776-777); y Córdoba Angulo, Miguel (2001, pp. 70-71). 
en los dos primeros por considerar que son expresión de un retorno a la legalidad y en el tercero por concluir lo contrario) ${ }^{12}$.

En el caso de las teorías psicológicas se excluyen por ende de antemano factores ajenos que se sobreponen a la voluntad del propio autor (teoría de la toma de decisión autoimpuesta). Para estos enfoques, para estimar satisfecho el requisito de la voluntariedad, es suficiente con que el agente se haya motivado per se, de manera completamente libre, más allá de los motivos o de las razones por las cuales interrumpió su plan criminal. Ejemplo de la jurisprudencia nacional, resuelto por la Sala Penal de la Corte mediante radicado 18.768: el actor sin más interrumpió el ataque que con un arma blanca y con intención de ocasionar la muerte ya había iniciado en contra de la víctima, oportunidad que esta última aprovechó para huir del lugar. Como en este caso no hubo ningún factor externo que haya provocado en el autor su decisión de interrumpir el ataque, habrá de aprobarse el requisito de la voluntariedad.

Con todo, como lo señala la postura crítica de la teoría psicológica de la toma de decisión autoimpuesta y en general de todas las teorías psicológicas, estas, por sí solas, terminan incurriendo en una especie de petición de principio. Pues en últimas no responden a la pregunta de en qué casos el autor ha desistido de forma autónoma o completamente libre. En terminología de Roxin, las teorías psicológicas que diferencian entre motivos autónomos y heterónomos son "inadecuada(s) porque se pueden vincular con ella significados tan diferentes que carece de todo contenido enunciativo autónomo" 13 , crítica que cabe igualmente a la postura que seguidamente se procede a analizar.

Autores como Frank han acudido a criterios de tipo corrector, complementario o de cierre para determinar este tipo de aspectos y dar solución a sus respectivos cuestionamientos. Según este autor (considerado como el creador de la "fórmula del motivo autónomo autodispuesto"), el desistimiento será voluntario cuando el sujeto que dio inicio a la ejecución del hecho ya no desea alcanzar la perfección de la conducta punible, a pesar de que tenía un ambiente y las condiciones completamente favorables para ello, por tener todavía un control pleno o el dominio total sobre el suceso. En este caso el motivo del desistimiento habrá sido completamente autónomo y por ello mismo libre de cualquier tipo de imposición. A contrapelo, no será voluntario el desistimiento cuando el autor ya no tenía estas condiciones a su favor por pérdida del control y sin embargo su deseo era alcanzar plenamente la consumación. En este otro hecho el motivo es heterónomo y por esa misma circunstancia está ausente el criterio de libertad de voluntad ${ }^{14}$.

12 Sobre las principales teorías de la voluntariedad del desistimiento, Roxin, Claus (Op. cit., pp. 714749), quien adhiere a las tesis normativas según se precisará más adelante.

13 Roxin, Claus (Op. cit., p. 744).

14 Sobre la concepción de Frank, Roxin, Claus (Op. cit., p. 743). 
La praxis judicial, por lo menos en el caso colombiano ${ }^{15}$, parece operar asimismo con criterios próximos a las teorías psicológicas. Por este camino se considera en los tribunales que la presencia de un móvil psíquico forzoso anula la voluntariedad, mientras que la existencia de alguna opción de elección en el autor produce los efectos contrarios. Así por ejemplo, en la decisión del 17 de julio de 2003, a la cual me he referido ya dando cuenta de su supuesto fáctico, la Corte Suprema de Justicia colombiana, Sala Penal, radicado 18.768, manifestó:

El desistimiento no puede tener su fundamento en circunstancias exteriores, ajenas a la voluntad del actor; puede surgir de un motivo autónomo, independientemente de que sea éticamente valioso (...) no habrá desistimiento si está provocado por impedimentos independientes de la voluntad del autor y que paralizan su voluntad de decisión (...).

Todas estas posturas psicológicas han sido cuestionadas por diversas razones en las que ahora no pretendo profundizar ${ }^{16}$. Sin duda, la crítica que en mi sentir se muestra con más fundamento es aquella que apunta a la imposibilidad empírica de comprobar la libertad de decisión. Como dicen Maurach, Gössel y Zipf:

Desde siempre se ha reprochado a esta concepción el que ella presupone una autodeterminación autónoma no comprobable del ser humano y un grado inconmensurable de libertad de cualquier coacción psíquica, con lo cual además de privilegiar al delincuente inescrupuloso, que no conoce la presión espiritual de intensos remordimientos de conciencia ${ }^{17}$.

Esta crítica sigue hoy vigente, sobre todo a partir de los estudios y los aportes tomados de la llamada neurociencia. Ciertamente, según los estudios de Libet, llevados a cabo en la década de los años ochenta del siglo pasado, el proceso de toma de decisión está precedido por el inconsciente, con lo cual existe un pequeñísimo lapso entre el estímulo y la consciencia de realización de una acción voluntaria. Esto ha servido para que algunos autores sostengan o quizá mejor nieguen la existencia de la libertad de voluntad (determinismo rígido). Sin embargo los estudios de Libet también permiten hablar -como de hecho lo ha desarrollado otro sector de la doctrina- en este mismo sentido de un indeterminismo débil, posición que comparto plenamente y en su totalidad, pues en últimas parece quedar claro que la actividad consciente está reservada para "bloquear" lo ya dispuesto por el inconsciente humano ${ }^{18}$. Lo

15 Igual sucede en el contexto alemán, según da cuenta de ello Roxin, CLAUS (Op. cit., pp. 714 y ss.), ilustrando al respecto con no pocos casos sometidos a consideración de los tribunales alemanes.

16 Cfr., sobre las críticas a las teorías psicológicas, Araque Moreno, Diego (2008, pp. 111-114).

17 Maurach, Gössel y ZiPf (1995, p. 104).

18 No es objeto de estudio del presente trabajo el problema de la demostración o negación de la libertad de voluntad. A tales efectos, el estudio más completo sobre este tema en el contexto nacional es el de 
anterior, dicho en otros términos, utilizando aquí una terminología prestada: cuando debo realizar una elección y no lo hago, esto es ya una elección.

Del otro lado se encuentran las teorías normativas. Dentro de estas destácase la de Jakobs (teoría de la modificación del hecho) y la de Roxin (teoría de las reglas de la profesión criminal). El primero entiende que el desistimiento es voluntario cuando el actor se muestra fiel al orden jurídico, lo cual se estima satisfecho cuando simplemente se separa o se aparta de la continuación del hecho teniendo todavía el control o el dominio de la situación, sin que exista ningún tipo de factor externo que modifique su empresa o propósito criminal. De acaecer esto último el desistimiento por el contrario no puede ser considerado como voluntario.

De acuerdo con la concepción aquí defendida -señala aquel-, el autor, que no necesita dominar con seguridad todo el acontecimiento, no tiene que realizar prestaciones especialmente meritorias, sino apartarse simplemente del hecho y esto ni siquiera de un modo final (...). Para la voluntariedad lo único que importa es que el autor se distancie del hecho ya puesto en práctica (...). De la misma forma en que falta ya la culpabilidad cuando el autor no está anímicamente dispuesto de un modo infiel hacia el Derecho (...) se da ya la voluntariedad cuando desaparece la infidelidad del autor hacía el Derecho en tanto no le haya sobrevenido un fracaso y tenga todavía plenamente en sus manos la evitación del resultado" 19 .

Roxin por su lado ha manifestado que hay lugar a reconocer los efectos liberadores del desistimiento si el actor, según una especie de reglas de la experiencia, desistió porque de forma inexplicable y completamente irrazonable lo invadió algún tipo de temor. En estos casos, desde el punto de vista valorativo, merece ser reconocido el efecto de impunidad que se sigue del desistimiento. Otra cosa deberá afirmase si el actor desistió por un cálculo serio y razonable ante la amenaza de ser descubierto. En este segundo evento no se merecen los efectos de la impunidad.

Cuanto más ‘curtido’ y ‘experimentado' sea el autor de una tentativa -señala Roxin-, tanto más se sentirá en condiciones de cometer el hecho pese a que exista un intenso peligro de ser descubierto. Pero también se retirará con tanta mayor cautela si el riesgo llega a ser demasiado grande -y precisamente entonces es involuntario el desistimiento-. Por otra parte, un delincuente relativamente inofensivo retrocederá ya lleno de miedo por un motivo insig-

Gómez Pavajeau, Carlos Arturo, quien asume una posición a favor del libre albedrío no estricto y moderado, y a donde remito precisamente al lector (2015, p. 58). Cfr., asimismo, con suficiencia, Fernández Carrasquilla, Juan (2013, pp. 140-155) y Pérez Manzano, Mercedes (2012, pp. 567-601).

19 JAKOBS, GÜNTHER (1997, pp. 344-345). 
nificante, aunque 'razonablemente' (es decir, según las reglas de la profesión criminal) habría podido seguir tranquilamente con su actividad; a este habrá que concederle la voluntariedad aunque internamente soportará una 'presión' mayor que el taimado bribón del caso paralelo ${ }^{20}$.

Tal parece que Roxin ha replanteado un poco su posición inicial. Pues hoy por hoy entiende desde su perspectiva normativa de los fines de la pena, y a partir de considerar este aspecto como el fundamento de la impunidad en los casos de desistimiento, que la voluntariedad de este debe ser valorada como tal en aquellos eventos en los cuales el autor ha retornado al camino o al sendero de la legalidad. Por ello en su criterio no se hace necesaria en estos casos la imposición de la pena. Y por esto mismo es que estima insostenible las posturas psicológicas, las cuales -en este orden de consideraciones- no tienen en cuenta el telos o la razón de ser de la figura jurídica del desistimiento en la consumación de la conducta punible. En sus propios palabras, "se impone cada vez más 'el topos rector' del 'retorno a la legalidad' o 'a la senda del Derecho""21.

A partir de este criterio Roxin ha confeccionado un grupo de casos en los cuales es posible sostener que el desistimiento efectivamente fue voluntario. Al primer grupo pertenecen aquellos sucesos en los cuales el autor desiste por motivos internos, eventos en los cuales, en mi sentir, simplemente no existe ningún tipo de coacción externa o ajena proveniente de un tercero (motivos loables, revisión de la decisión, cargos de consciencia, vergüenza interna, razonamiento pausado, sentimientos de compasión, falta de fuerza en la decisión, sentimientos de miedo, pérdida de interés, insatisfacción personal, falta de ganas, entre otros). Aquí tendría cabida por ejemplo el caso resuelto por la jurisprudencia colombiana al que se hizo referencia con anterioridad. El autor que sin más cesa en su ataque criminal, ha desistido por motivos internos que incluso en muchos de los casos solamente él conoce. Con ello es suficiente, según Roxin, para valorar positivamente su retorno al camino de la legalidad y por tanto no se hace necesaria la imposición de la pena. En el segundo grupo se aglutinan los eventos en que el autor se ve compelido a desistir por causas externas que no aumentan -o si lo hacen resultan de poca monta-el riesgo de fracaso o posterior exposición a la sanción penal (circunstancias que simplemente estorban la realización del hecho, horror causado por el estado de la víctima, súplicas, reproches o persuasiones del sujeto pasivo, motivos religiosos o supersticiones causados por factores externos, etc.).

El hecho que le sirve a Roxin para explicar este otro grupo es el siguiente: el sujeto apuñaló a su esposa con intención de ocasionarle la muerte. Intempestivamente hicieron presencia en el lugar, la habitación de la pareja, los hijos de esta. El sujeto

20 Roxin, Claus (1976, p. 254).

21 Roxin, Claus (2014, p. 722). 
declinó en consecuencia de su delito por la presencia de los hijos y porque emocionalmente no era capaz de continuar con su ejecución en su presencia. Situación que persistió con posterioridad cuando habida cuenta la proximidad echó a los menores del lugar y de nuevo quedó a solas con su mujer. Aquí, según este autor, el sujeto de todas formas ha regresado al camino del respeto por el Derecho, por lo cual está a favor de la voluntariedad y en contra de la negativa que sería sostenida por las posiciones psicológicas. En este segundo grupo, de forma personal considero simplemente que el factor externo no es de tipo insuperable, razón por la cual el autor se puede perfectamente sobreponer al mismo, de suerte que también estoy a favor de aprobar la voluntariedad.

Finalmente, en lo que tiene que ver con la llamada lógica del delincuente, por él propuesta, Roxin señala que quizá su posición inicial fue malinterpretada, pues con esto solo quería decir en su momento que dicho criterio trata "de un principio normativo que debe ayudar a determinar qué puede considerarse como retorno a la legalidad, contrario al plan"22. De esta manera estima responder a las críticas que en su momento le fueron formuladas.

De los cuestionamientos a estas posturas normativas ya me ocupé en su momento, por lo que remito a lo ya dicho en su lugar ${ }^{23}$.

Por su lado, Restrepo Ramos también encuadra su propuesta dentro de los enfoques normativos, según se tendrá oportunidad de examinar infra, el cual ciertamente me parece favorable en los términos valorativos o de interpretación de cada caso y según la postura que desarrollaré más adelante, pero que desde ya acude al criterio de la coacción como causa de inculpabilidad penal para aplicar sus reglas al requisito de la voluntariedad en la consumación del delito y así concretar la ambigüedad que se advierte por algún sector de la doctrina, teniendo como punto de partida el enfoque de las tesis psicológicas (postura mixta). Como lo manifesté en otra ocasión, se trata de establecer aquellos aspectos que "permitan valorar que la interrupción del fenómeno delictivo no proviene de la imposición de circunstancias o variables que anulen la propia autonomía del agente, que dobleguen su voluntad, sin que desde luego para nada importe el tema de la espontaneidad" 24 .

Otros autores como Muñoz Conde sugieren igualmente partir de una teoría mixta compuesta por un criterio psicológico -en donde se analiza si el sujeto todavía estaba en condiciones de seguir actuando y si abandonó concretamente su propósito de consumación- y la respectiva valoración de los motivos que tuvo para ello, amén

22 Roxin, Claus (Op. cit., p. 725).

23 Araque Moreno, Diego (2008, pp. 115-120).

24 Araque Moreno, Diego (2008, p. 122). 
del análisis de la situación a la luz de los fines de prevención general y especial de la pena ${ }^{25}$.

\section{HIPÓTESIS: LA VOLUNTARIEDAD DEL DESISTIMIENTO COMO EXPRESIÓN DE AUSENCIA DE COACCIÓN}

En mi trabajo de 2008 consideré que la voluntariedad como primer requisito del desistimiento debía ser entendida como ausencia de coacción, sin importar en lo absoluto la valoración de los motivos que tuvo en cuenta el autor a la hora de retroceder en su impulso criminal objetivado en los actos de ejecución del punible. Un ejemplo más para ilustrar este aserto, tomado de nuevo de Roxin, quien a partir de su postura normativa de regreso al camino del Derecho afirma involuntariedad: El autor, luego de haberse confabulado con el vendedor y de llevar a cabo conjuntamente actos de engaño orientados a cobrar un seguro, a cambio del pago de una suma de dinero, le dice luego a la aseguradora que el vendedor ha participado en el respectivo engaño (motivo interesado). Como en su momento lo expuse $-\mathrm{y}$ en esto coincide la mayoría de la doctrina y la jurisprudencia- debe tenerse como punto de arranque el hecho de que pueden perfectamente ser consideradas como voluntarias razones que no se compadezcan en lo más mínimo con el reforzamiento de valores éticos, dignos de buen ejemplo, valiosos o virtuosos. Aspectos que, en un modelo de derecho penal de acto, no pueden constituir ningún tipo de obstáculo para reconocer los efectos liberadores de pena que se siguen de la figura jurídica del desistimiento. Esto último, desde luego, siempre y cuando se cumpla adicionalmente con el requisito de la evitación o eficacia. Considerar lo contrario, esto es, que la voluntariedad en el desistimiento está condicionada a un motivo éticamente irreprochable, es tanto como confundir el derecho con la moral, el delito con el pecado, el derecho penal de autor con el derecho penal de acto. En suma, de entrada no importa la valoración del móvil por el cual desistió el autor. Con lo cual es voluntario el desistimiento por razones interesadas, de satisfacción personal, remordimiento, aplazamiento, oportunidad, lástima y humillación, entre otras.

Lo anterior sin embargo poco o nada dice con miras a determinar el problema de fondo, es decir, entrar a valorar si el desistimiento de la consumación del injusto es o no voluntario. Por consiguiente es indispensable proceder en segundo plano a establecer si la interrupción delictiva por parte del propio autor se explica por la imposición de factores externos que en consecuencia afectan su capacidad de autodeterminación, su libertad de voluntad, caso en el cual el desistimiento no podrá ser estimado como voluntario; por el contrario, sino concurre ningún factor externo de

25 Muñoz Conde, Francisco (1972, pp. 103 y ss.). Asimismo, autores como Stratenwerth, Günter (2005, pp. 304-306) consideran que los criterios psicológicos y normativos por sí solos resultan insuficientes. 
este tipo (como por cierto y en efecto acontece en el ejemplo que acabo de reseñar), si el agente abandonó su idea criminal de forma completamente autónoma, con libertad de voluntad, el desistimiento de llevar a cabo la conducta punible deberá ser valorado como voluntario, con lo cual quedará satisfecha esta primera exigencia.

Sobre este otro punto continúo sosteniendo lo que en su momento señalé al respecto, valga recordar, que es necesario para estos efectos que el autor como prerrequisito todavía tenga el control o el dominio de la situación. Pues, de lo contrario, en caso de haber perdido de forma definitiva el dominio sobre el suceso, ya no es posible hablar de voluntariedad sino más bien de un fracaso y por consiguiente de un acto de arrepentimiento. Pero no basta simplemente con este dato. En mi sentir, se requiere además que el sujeto, teniendo el control pleno del suceso como ya lo señalé, abandone su consumación sin que obre sobre él ningún tipo de coacción. El desistimiento, en estos casos, conforme al uso corriente del lenguaje y de las palabras, se explicará por razones que únicamente son imputables a la autonomía del propio autor. Habrá sido él mismo quien desactivó el riesgo creado para el respectivo bien jurídico. Si en cambio se trata de razones que le fueron impuestas, el sujeto habrá sido coaccionado o constreñido a abandonar el plan criminal y por ello mismo no podrá hablarse de voluntariedad pues el desistimiento no responderá en este otro caso a su libre decisión. Citando en este punto nuevamente a Maurach, Gössel y Zipf, "para constatar la voluntariedad solo podemos atender por ahora a la libre autodeterminación por motivos autónomos"26.

Dentro de este concepto de autonomía, como también ya lo expuse, caben perfectamente razones como el miedo genérico o abstracto a la pena (derivado de los efectos de amenaza de la sanción penal en el proceso de selección primaria de las conductas que le interesan al legislador con miras a desestimular su realización), sentimientos de piedad por la víctima, aplazamiento de la ejecución para otra ocasión más propicia, concentración de sus esfuerzos en otra víctima, motivos de satisfacción personal, razones de pudor propio o incluso reproches de un tercero. En estos ejemplos es claro a mi juicio que, según el uso corriente de las palabras, la persona declinó de sus planes ilícitos iniciales con plena autonomía, con capacidad libre de decisión o de voluntad. Por ello la no consumación de la conducta punible se explica en términos valorativos por factores o motivos que únicamente pueden ser atribuidos a su propia voluntad, si bien es cierto que algunos de ellos pueden provenir del exterior.

Dicho en otras palabras, lo anterior significa que para determinar la voluntariedad del desistimiento es perfectamente posible acudir a las reglas que rigen la coacción en materia de inculpabilidad penal, mismas que así vistas las cosas permitirán valorar en el caso concreto el problema de la voluntariedad. En los casos de insuperable coacción ajena como es sabido se considera que no hay responsabilidad penal por 
ausencia del componente de la culpabilidad. La persona que obra ilícitamente bajo los efectos de una coacción insuperable lleva a cabo un injusto penal pero falta en ella el presupuesto de la culpabilidad. El acto de coacción en estos casos es entendido generalmente como un constreñimiento que, proviniendo de un tercero, obliga al sujeto a realizar u omitir algo en contra de su voluntad. Por esta razón se ve afectada su libertad de autodeterminación y en consecuencia se considera que la persona no ha obrado con capacidad de culpabilidad penal ${ }^{27}$.

Según la jurisprudencia colombiana:

La insuperable coacción ajena se origina en la acción de un tercero que constriñe la voluntad de otro mediante violencia física o psíquica (o moral), para que ejecute un comportamiento típico de acción o de omisión que sin tal sometimiento no realizaría; en otras palabras, el sujeto activo no goza de las condiciones para gobernar a plenitud su voluntad ya que su libre autonomía está dominada por la compulsión del coaccionador. En esta causal se configura, en primer término, la acción injusta e intencional de quien coacciona para someter a otro, y en segundo, la reacción psíquica del doblegado quien padece los efectos emocionales de la coacción, merced a la cual comete el hecho típicamente antijurídico sin reflejar en él un acto de su verdadera voluntad o su espontaneidad, la exoneración de la culpabilidad se afianza, no en la supresión absoluta de la voluntad, sino en la reducción del ámbito de la libre autodeterminación ${ }^{28}$.

Por lo tanto, así como en los casos de coacción insuperable falta entonces la libertad de decisión como presupuesto de la culpabilidad penal por efectos del factor constrictivo, en los casos del desistimiento faltará la voluntariedad en todos aquellos eventos en los cuales el sujeto desista de su ilícito propósito por factores externos que, proviniendo de otro u otros sujetos -incluida la víctima, claro está- o cualquier otro tipo de circunstancia -una cámara de vigilancia, por ejemplo- lo constriñen, lo obligan o lo compelen a ello en el buen sentido de las palabras. En estos eventos por lo tanto el desistimiento no se explica por razones atribuibles al propio autor. No podrá imputarse la evitación del resultado a su propia voluntad. Antes bien, su explicación se remite a factores impuestos que afectan su libertad de autodeterminación y que permiten valorar que no fue él quien desactivó el riesgo jurídicamente desaprobado que previamente incrementó en relación con el respectivo bien jurídico. De esta forma, en mi sentir, se concreta la indeterminación general que se cuestiona a las propuestas psicológicas.

27 Sobre la insuperable coacción ajena como causa de inculpabilidad penal, véase, ARAQUE MoRENO, DiEGo (2016, pp. 349-350).

28 Sentencia de 22 de julio de 2009, radicado 27.277. 
De hecho, con arreglo a esta concepción, el desistimiento puede ser voluntario pese a no ser espontáneo por ser sugerido por ejemplo por otra persona -incluida la víctima-, siempre y cuando no sea definitivamente impuesto por razones que afecten la libertad de voluntad según las reglas generales del uso del lenguaje. V.gr., es voluntario el desistimiento cuando la esposa, luego de suministrar un potente veneno a su marido, obviamente con intención de quitarle la vida, llama a un médico como consecuencia de las insistentes súplicas de aquel, lográndose de esta manera la evitación del resultado (caso de la jurisprudencia alemana).

En esto, comparto lo afirmado por algún sector de la doctrina que examina la voluntariedad del desistimiento a partir de determinar los supuestos de coacción acudiendo a las reglas del estado de necesidad. Después de todo es claro que los casos de coacción insuperable son vistos por algunos autores como eventos de inexigibilidad penal. Por este camino se llega a la conclusión de que la coacción es un evento más de estado de necesidad exculpante. El resultado es el mismo independientemente del camino escogido -coacción, inexigibilidad o estado de necesidad exculpante-. Si la razón por la cual el sujeto desistió aplicara como candidata a ser considerada como una causa de inculpabilidad penal, el desistimiento debe en estos eventos por equivalencia considerarse como involuntario. En sentido contrario, si la explicación del desistimiento en el caso concreto no aplica como candidata a ser considerada como una causa de inculpabilidad penal en los términos anteriormente referidos, entonces el desistimiento efectivamente ha de ser tenido como plenamente voluntario por equiparación. En sentido próximo, en la doctrina española, si creo entender, se desarrolla el problema por Pozuelo Pérez, quien en todo caso estima que la valoración del hecho se debe llevar a cabo dependiendo en la coacción -la cual estima como una subespecie de estado de necesidad- de la magnitud de la amenaza, su probabilidad de acaecimiento y la situación o características del sujeto sobre el cual recae ${ }^{29}$.

En Alemania Jäger ha propuesto acudir a las reglas de la autoría mediata para efectos de negar la voluntariedad en aquellos casos en los cuales el sujeto ha obrado por amenaza, inimputabilidad provocada por sí mismo (ejemplos de actio liberae in causa), error del instrumento y del cual se vale el sujeto de atrás, y pérdida del sentido de la acción. En estos supuestos, en su sentir, la voluntad no se ha configurado de forma plenamente libre en el sujeto que es actuado y por ello mismo no puede ser imputada a este ${ }^{30}$. Dicha posición, según Roxin, ha sido recientemente matizada por el autor acudiendo a los casos de exculpación por estado de necesidad consagrados en el parágrafo 35 del $\mathrm{StGB}^{31}$. Por mi parte considero que nuestra legislación permite llegar parcialmente a similares conclusiones, sin acudir a la figura de la autoría

29 Pozuelo PÉrez, Laura (2003, pp. 259 y ss.)

30 JÄGER, CHIRSTIAN (2003, pp. 17-44).

31 Véase, sobre la reciente posición de JÄGER y sus respectivas críticas, RoXIN, CLAUS (2014, pp. 747749). 
mediata, ello es, por intermedio de las reglas que configuran la llamada insuperable coacción ajena y que se consagra en el numeral 8 del artículo 32 del Código Penal.

\section{IV. ¿DESISTIMIENTO VOLUNTARIO O "VÁLIDO”?}

En el apartado anterior expuse las razones por las cuales creo que el desistimiento puede ser interpretado como un acto voluntario atribuible a factores que se explican a la autonomía del propio autor, a su libertad de decisión o de voluntad, aplicando las reglas de la coacción insuperable con el fin de concretar su valoración, dándose así por satisfecho el primer requisito para efectos de otorgar las consecuencias de exoneración de responsabilidad penal propias del retroceso criminal. En un trabajo reciente Restrepo Ramos considera en cambio que no es posible acudir en estricto rigor a la voluntariedad como primer requisito. En su lugar propone determinar en qué casos el desistimiento es válido, acudiendo a una fundamentación de razón práctica. En dicho trabajo, luego de analizar el fundamento de la impunidad del desistimiento y su ubicación sistemática dentro de la teoría del delito, se ocupa del problema en concreto de la voluntariedad y de las principales teorías que se han elaborado al respecto, algunas de las cuales fueron tratadas con anterioridad.

En su opinión, el concepto de voluntariedad puede ser entendido como un acto de decisión libre o porque confluye alguna razón que en nada afecta a la autonomía ${ }^{32}$. A partir de aquí considera que toda actuación humana es necesariamente voluntaria y de allí que no es posible diferenciar entre los distintos casos posibles. En las propias palabras de Restrepo Ramos:

Una fórmula que simplemente estime como voluntario el comportamiento que "pudo haber sido de otra forma" tiene que decir que todo comportamiento es "voluntario", con lo cual "ni siquiera en los casos de coacción podría establecerse que hubo desistimiento voluntario: al analizar su razonamiento práctico, quien es víctima de la llamada coacción "insuperable" siempre puede optar por sufrir con las consecuencias con las que se le amenaza.

Este aserto, como resulta fácil advertirlo, va en contra de la tesis que sostengo. Por este razonamiento, considera entonces que la voluntariedad como requisito del desistimiento no puede agotarse en que haya existido en la toma de decisión alguna razón para actuar, por lo que en su lugar se haría necesario establecer si la razón respectiva es o no "válida" 33 y partir en consecuencia de una teoría normativa en materia de fundamento del desistimiento del delito tentado que estima se edificaría

32 Restrepo Ramos, Jorge C. (2015, p. 167).

33 Restrepo Ramos, Jorge C. (Op. cit. 168-169). 
en la legislación colombiana a partir del principio de legalidad y la redacción del artículo 27 , así como el principio de motivación indirecta de la ley penal ${ }^{34}$.

En este punto discrepo de lo afirmado por el autor. En efecto, en mi criterio la voluntariedad del desistimiento consiste no en establecer si las razones que lo motivaron fueron válidas o no a la luz del ordenamiento jurídico que regula los contactos intersubjetivos en cada uno de los Estados. Opino, como ya lo expresé, que la voluntariedad, a partir del uso corriente de las reglas del lenguaje, puede ser determinada y concretada en este contexto como un acto de libre voluntad del autor, como libertad de autodeterminación, como expresión de su propia autonomía, independientemente de las razones que lo motivaron y desde luego de la validez o invalidez de estas últimas. A tales efectos, he considerado y sigo haciéndolo que para establecer dicho aspecto se puede acudir a las reglas de la coacción que rigen en materia de inculpabilidad penal. Si el sujeto desistió porque fue constreñido a ello por algún factor externo, su desistimiento no podrá ser estimado como voluntario toda vez que en este sentido este último no obedeció a un acto de su libre decisión o autonomía sino que por el contrario el mismo le fue impuesto. Así como en los casos de vis compulsiva, cuando esta es insuperable, se excluye la culpabilidad penal o responsabilidad subjetiva por el hecho cometido, en los eventos en que el sujeto desista por verse coaccionado, constreñido o compelido a ello se excluirá el requisito de la voluntariedad en materia de desistimiento por equiparación, razón por la cual se impone la sanción por un delito tentado. En sentido opuesto, si el factor por el cual desistió el autor no aplica como causa de exoneración de la culpabilidad penal por no configurar una coacción de tipo insuperable, el desistimiento en estos casos, también por equiparación, deberá ser valorado como voluntario. Perfectamente podría suceder que en un caso en concreto la razón o el motivo por el cual se desiste no sea "válido" y sin embargo sí pueda ser interpretado como voluntario. Esto puede ser así porque las teorías normativas, por más que se sostenga lo contrario ${ }^{35}$, terminan contradiciendo el principio de legalidad de delitos y de penas. Lo que se exige es voluntariedad, no validez, criterio este que resulta bastante anfibológico y por tanto susceptible de fácil manipulación por parte del operador jurídico. Por ello mismo, se erige en una llave que abre la compuerta a la arbitrariedad. Paradójicamente, por este camino, no se respeta el principio de legalidad que como punto de partida asume Restrepo Ramos en su elaboración conceptual. Antes bien, termina sucediendo todo lo contrario, se soslaya abiertamente el principio de la referencia.

No se trata entonces de analizar si la razón o el motivo en sí mismo que determinó el acto de decisión de desistir es válido o inválido para efectos de dar como establecida la exigencia de la voluntariedad como requisito del desistimiento. Esto, insisto, sería contrario al tenor literal del artículo 27 del Código Penal colombiano

34 Restrepo Ramos, Jorge C. (Op. cit., p. 170).

35 Roxin, Claus (2014, pp. 733-736). 
y a su interpretación sensu contrario en materia de desistimiento penal. Se trata en su lugar de determinar si el acto de decisión en sí mismo considerado obedeció a una decisión libre tomada por el propio autor o en cambio fue provocado por una circunstancia que, afectando su libertad de decisión, provocó en él la resolución de desistir de la consumación del delito. Cambiando lo que se debe cambiar, aplicando a estos casos las reglas de la coacción de carácter insuperable que rigen en materia de inculpabilidad penal, es posible establecer por equivalencia si fue voluntario o no el desistimiento del propio autor en el sentido de no persistir en la realización de la conducta punible.

Lo anterior no quiere decir que se esté asimilando el concepto de voluntariedad con el de capacidad de decisión. Más bien significa que la voluntariedad para efectos del desistimiento en materia de derecho penal está relacionada con la libertad en la toma de decisiones, la cual se puede ver reducida obviamente en casos de coacción que se traducen en materia de desistimiento en la desaprobación del componente de la voluntariedad. Desde luego que quien actúa bajo amenaza toma una decisión de obrar. En esto asiste la razón a Restrepo Ramos. Pero esta no fue una elección libre del agente, de su propia autodeterminación, motivo por el cual no se explica por razones autónomas sino completamente impuestas. He aquí la explicación por la cual, para los efectos del desistimiento, no puede ser tenida como voluntaria. Al efecto, Martínez Escamilla, quien parte de una postura psicológica en la doctrina española, señala lo siguiente, en observación que comparto plenamente:

Imaginemos una persona que es obligada a entregar el dinero de la caja fuerte bajo la amenaza de lesiones. Objetivamente nadie niega la existencia de una alternativa con dos opciones: dejarse lesionar o entregar el dinero. Aunque el sujeto mantuviese la frialdad de ánimo suficiente para ponderar los pro y contras de ambas posibilidades, decidiéndose por la segunda, no creo que nadie se atreviera a afirmar que la persona de nuestro ejemplo ha entregado voluntariamente el dinero exigido. Lo mismo ocurre en los casos de desistimiento"36.

De todas maneras Restrepo Ramos estima que las razones coactivas no pueden ser valoradas como un motivo válido de razón para actuar a efectos de tener como voluntario el desistimiento. Es esta una segunda regla de su elaboración conceptual, con la cual coincido plenamente en sus resultados, al igual que con las restantes. La primera regla apunta a que el desistimiento no exige que los móviles sean valorados como correctos, buenos o cualquier cosa que se le parezca. La tercera consiste en que el temor abstracto a la pena ha de ser valorado como una razón válida de desistimiento por no poderse hablar en este caso en sentido estricto de coacción. La cuarta regla

36 Martínez Escamilla, Margarita (1997, p. 334). 
elaborada por el autor, con arreglo al principio del acto, consiste en que será equivalentemente válido el desistimiento cuando la razón en sí es inválida putativamente. A mi consideración, si el sujeto desistió porque erradamente creyó que había sido descubierto en la realización de su plan criminal, cosa que en realidad no ha sucedido, en verdad no ha existido ningún acto externo de coacción o de constreñimiento que afecte su libertad de decisión o de voluntad. Aquí el desistimiento es voluntario sin más, por ausencia real de coacción, y no por simple equivalencia como en estos casos lo propone el autor ${ }^{37}$. Un último ejemplo, esta vez del propio Restrepo Ramos: el actor cree que ha sido descubierto por la fuerza pública que hizo presencia en el lugar, luego de entrar a un supermercado y tomar algunos enceres para apoderarse de ellos; en realidad, los uniformados no se habían percatado de la situación. Como lo acabo de manifestar, aquí no existe coacción real y por lo mismo apruebo en este caso la voluntariedad. En el peor de los casos, el sujeto habrá creído que desistió por coacción, cosa que en verdad no fue así, no fue cierta. Por equiparación entonces, si se quiere, deberían aplicarse las reglas del error de prohibición indirecto sobre los presupuestos objetivos de una causa de ausencia de responsabilidad penal (numeral 10 , artículo $32 \mathrm{CP}$ ) y examinarse su invencibilidad o vencibilidad para efectos de su posible exoneración.

\section{BIBLIOGRAFÍA}

Araque Moreno, Diego (2008). Desistimiento y Derecho penal. Bogotá: Ibáñez.

Araque Moreno, Diego (2009). “Contribución al estudio sobre el fundamento del desistimiento en derecho penal”, en Derecho penal contemporáneo, n. ${ }^{\circ} 29$. Bogotá: Legis, pp. 93-109.

Araque Moreno, Diego (2011). “Tentativa”, en Aa/vv, Derecho penal. Parte general. Fundamentos. Medellín: Sello Editorial Universidad de Medellín.

Araque Moreno, Diego (2016). Lecciones de Derecho penal. Introducción y fundamentos de imputación de responsabilidad penal. Bogotá: Ibáñez.

Córdoba Angulo, Miguel (2001). La tentativa. Bogotá: Universidad Externado de Colombia.

Córdoba Angulo, Miguel (2002). “Tentativa”, en Aa/vv, Lecciones de Derecho penal. Parte general. Bogotá: Universidad Externado de Colombia, pp. 289-300.

37 Sobre las reglas elaborados por RESTREPO RAmOs (Op. cit., pp. 171-177). 
Fernández Carrasquilla, Juan (2012). Derecho penal. Parte general. Teoría del delito y de la pena. Vol. 2. Dispositivos amplificadores, concurso y penal. Bogotá: Ibáñez.

FERnÁNDEZ CARrasquilla, JuAn (2013). "Culpabilidad y libertad de voluntad", en Anuario de Derecho penal y ciencias penales, Vol. LXVI. Madrid, pp. 140-155.

Gómez López, Jesús Orlando (2003). Teoría del delito. Bogotá: Ediciones Doctrina y Ley.

Gómez Pavajeau, Carlos Arturo (2015). Neurociencias y Derecho. Bogotá: Ediciones Nueva Jurídica.

JÄGER, CHIRSTIAN (2003). "El elemento volitivo en el desistimiento de la tentativa. Aportación para la asimilación de las Teorías de Autoría y el Desistimiento", en Anuario de Derecho penal y ciencia penales, T. LVI. Madrid: ADPCP, pp. 17-44

JAKOBS, GÜNTHER (1997). "El desistimiento como modificación del hecho", en Estudios de Derecho Penal. Traducción de EnriQue Peñaranda Ramos, Carlos J. Suárez González y Manuel Cancio Meliá. Madrid: Ediciones Universidad Autónoma de Madrid - Editorial Civitas, pp. 325-345.

Jescheck, Hans-Heinrich y Weigend, Thomas (2002). Tratado de Derecho penal. Parte general.5. a ed. Traducción de Miguel Olmedo CARdenete. Navarra: Comares.

Martínez Escamilla, Margarita (1997). "Dos cuestiones básicas del desistimiento en Derecho penal", en Política criminal y nuevo Derecho penal. Libro Homenaje a Claus Roxin. Barcelona: Bosch Editor, pp. 331-340.

MAUrach, Reinhart; Gössel, KARL h. y ZIPF, heINZ (1995). Derecho penal. Parte general 2. Formas de aparición del delito y las consecuencias jurídicas del hecho.

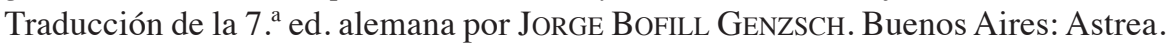

Muñoz Conde, Francisco (1972). El desistimiento voluntario de consumar el delito. Barcelona: Bosch.

Pérez Manzano, Mercedes (2012). "El tiempo de la consciencia y la libertad de decisión: bases para una reflexión sobre Neurociencia y responsabilidad penal”, en Diego Araque (Coord.). Estudios de Derecho penal. Libro homenaje a Juan Fernández Carrasquilla. Medellín: Sello Editorial Universidad de Medellín.

POZUelo PÉrez, LAURA (2003). El desistimiento en la tentativa y la conducta postdelictiva. Valencia: Tirant lo blach. 
RESTREPO RAMOS, JoRgE C. (2015). "Esbozo de cuatro reglas sobre la voluntariedad del desistimiento de la tentativa", en Revista Derecho Penal y Criminología, Vol. 36, n. ${ }^{\circ}$ 101, julio-diciembre de 2015, Bogotá: Universidad Externado de Colombia, pp. 153-179.

Reyes Alvarado, Yesid (2016). El delito de tentativa. Montevideo-Buenos Aires: Editorial B. de F.

Roxin, Claus (1976). "Sobre el desistimiento de la tentativa inacabada", en Problemas básicos del derecho penal. Traducción de Diego-Manuel LuZÓn PEÑA. Madrid: Reus.

Roxin, Claus (2014). Derecho penal. Parte general. Tomo II. Especiales formas de aparición del delito. Traducción y notas de Diego-Manuel Luzón Peña, José MAnuel Paredes Castañón, Miguel Díaz y García Conlledo y Javier de Vicente REMESAL. Navarra: Thomson Reuters-Civitas.

Stratenwerth, GÜnter (2005). Derecho penal. Parte general I. El hecho punible. Traducción de Manuel Cancio Meliá y Marcelo A. Sancinetti. Navarra: Thomson-Civitas.

Velásquez V., Fernando (2009). Derecho penal. Parte general. 4. a ed. Medellín: Comlibros. 